\title{
Use, and perceived usefulness, of cognitive behavioural therapy techniques for self-care among therapists
}

\author{
Gerhard Andersson ${ }^{1,2}$ (D) Andreas Björklind ${ }^{1}$, James Bennett-Levy ${ }^{3}$ (D) and Benjamin Bohman ${ }^{2 * *}$ (D) \\ ${ }^{1}$ Department of Behavioural Sciences and Learning, Linköping University, Linköping, Sweden, ${ }^{2}$ Centre for Psychiatry \\ Research, Department of Clinical Neuroscience, Karolinska Institutet and Stockholm Health Care Services, Region \\ Stockholm, Stockholm, Sweden and ${ }^{3}$ University Centre for Rural Health, The University of Sydney, Lismore, Australia \\ ${ }^{\star}$ Corresponding author. Email: benjamin.bohman@ki.se
}

(Received 15 July 2020; revised 31 August 2020; accepted 1 September 2020)

\begin{abstract}
Mental health problems are prevalent among therapists and may have a negative impact on therapist effectiveness. To counteract such problems, therapist self-care (for example, striking a balance between personal and professional demands and seeking personal therapy), has received increased attention. Conceptually, self-care can be considered as part of a personal practice model, focusing on techniques that therapists engage with self-experientially with a focus on their personal and/or professional development. However, studies of the self-application of specific treatment techniques are lacking. We aimed to explore the use, and perceived usefulness, of cognitive behavioural therapy (CBT) techniques for self-care to prevent or treat own mental health problems among practising therapists. Participants were therapists $(n=228)$ of various professional backgrounds in Sweden. Data were collected using a web-based survey. Descriptive statistics were calculated, and non-parametric analyses conducted to investigate associations of 13 CBT techniques with therapist characteristics. Use of CBT techniques for self-care was highly prevalent among participants, and they perceived the techniques as useful, irrespective of characteristics such as gender, age, profession, years since graduation, clinical experience, level of training in CBT, and previous experience of personal CBT. The high prevalence among therapists of the use of treatment techniques for self-care is very encouraging. Therapist selfcare, including the self-application of treatment techniques, may be an important factor for therapist effectiveness, which calls for further development of personal practice models with respect to self-care, and future studies investigating associations between therapist mental health, self-care, effectiveness and patient outcome.
\end{abstract}

\section{Key learning aims}

(1) Therapist self-care using cognitive behavioural therapy (CBT) techniques to prevent or treat own mental health problems may influence therapist effectiveness. However, studies of self-application of treatment techniques are lacking.

(2) In the present survey study, the use of CBT techniques for self-care was highly prevalent among practising therapists, and they perceived the techniques as useful, irrespective of characteristics such as gender, age, profession, years since graduation, clinical experience, level of training in $\mathrm{CBT}$, and previous experience of personal CBT.

(3) Almost all therapists believed that it was a good idea to self-apply CBT techniques for their own sake and for the benefit of their patients.

Keywords: cognitive behavioural therapy; mental health; self-care

\footnotetext{
() British Association for Behavioural and Cognitive Psychotherapies 2020. This is an Open Access article, distributed under the terms of the Creative Commons Attribution licence (http://creativecommons.org/licenses/by/4.0/), which permits unrestricted re-use, distribution, and reproduction in any medium, provided the original work is properly cited.
} 


\section{Introduction}

Mental health problems are prevalent among psychologists and other therapists working in psychiatric health care services. In a recent survey of 678 clinical psychologists in the UK (Tay et al., 2018), 63\% reported having experienced self-defined mental health problems at some point in their lives, particularly anxiety and depression. This compares with a $43 \%$ lifetime prevalence of mental health problems in the general adult population in the UK (Mental Health Foundation, 2016). Furthermore, in a survey of 425 counselling psychologists in the USA (Gilroy et al., 2002), 62\% self-identified as depressed, and in another USA survey of psychologists (American Psychological Association, 2010), 18\% of 602 respondents reported they had had suicidal ideation while dealing with personal and professional stressors or challenges. In a recent systematic review involving 8808 therapists, primarily psychologists, the prevalence of moderate to high work-related stress and burn-out was 55\% (Simionato and Simpson, 2018). Mental health problems in therapists may have a negative impact on therapist effectiveness, including an inability to maintain focus with patients, memory problems, fatigue, and lack of energy and motivation for therapeutic work, which can lead to boundary and other ethical violations (American Psychological Association, 2010; Gilroy et al., 2002). Several factors have been suggested as contributing to mental health problems in therapists working in psychiatric health care services. Among these factors are high emotional demands and stresses inherent in working with patients in this field, an isolating work situation with limited contact with colleagues and confidentiality obligations, a 'professional blind spot' with regard to own mental health problems due to being trained to attend to others' problems, and those with lived experience may be more likely to be attracted to this line of work (Barnett and Cooper, 2009). In addition, stigma, shame and concerns about negative consequences for career may prevent disclosure and help-seeking (Tay et al., 2018), which may further exacerbate mental health problems.

Not surprisingly, ethics codes of many professional organizations include statements of therapist awareness of own health issues and their possible effects on patients, and what actions should be taken. For example, the ethics code of the American Psychological Association (2002) states that psychologists should refrain from professional activity if personal problems may prevent them from performing in a competent manner, and that they should take appropriate measures, such as seeking professional consultation. Similarly, the ethical code of the European Federation of Psychologists' Associations (2005) includes an obligation not to practise when ability or judgement is adversely affected, for example due to temporary personal problems. Another example is the Health and Care Professions Council in the UK, which promotes 'professional self-regulation' and states in its standards of proficiency for psychologists (Health and Care Professions Council, 2015) that they must be able to maintain fitness to practise by understanding the importance of maintaining their own health, and to manage the physical, psychological and emotional impact of their practice.

To counteract mental health problems and comply with ethical standards, self-care of therapists has received increased attention (Barnett et al., 2007). Common preventative selfcare strategies recommended for psychologists and other therapists include striking a balance between personal and professional demands, taking regular breaks from work, getting adequate rest and exercise, having a healthy diet, attending to emotional, relationship and spiritual needs outside of work, and seeking assistance in the form of peer support and supervision (Barnett et al., 2007; Barnett and Cooper, 2009). Common corrective self-care strategies among therapists include meditation (e.g. mindfulness and compassion; Boellinghaus et al., 2013; Lomas et al., 2019) and personal therapy (Orlinsky et al., 2011). In the aforementioned survey of psychologists in the USA (American Psychological Association, 2010), the top three of the ten most frequently reported self-care strategies were maintaining a balance between personal and professional lives (96\%), seeking support from friends or peers 
(95\%), and talking to a colleague (94\%). Receiving psychotherapy or counseling ranked eighth and was reported by $64 \%$ of respondents. Despite being naturally accessible to therapists and recommended as self-care strategies (Norcross, 2000), less attention has been paid in the selfcare literature to the self-application of specific treatment techniques, such as cognitive restructuring, exposure and applied relaxation. Thus, it is not known to what extent practising therapists use treatment techniques on themselves and how they perceive them.

Conceptually, self-care can be considered as part of a personal practice (PP) model. PP has been defined as 'formal psychological interventions and techniques that therapists engage with selfexperientially ... with a reflective focus on their personal and/or professional development' (Bennett-Levy, 2019; p. 133). There are several different forms of PP and the emphasis on personal or professional development varies. Three of the most common forms are personal therapy, meditation programmes and self-practice/self-reflection programmes (Bennett-Levy, 2019); the latter refers to therapists practising treatment techniques on themselves and reflecting on the experience, primarily with a professional focus on developing skills. According to the PP model developed by Bennett-Levy and Finlay-Jones (2018), there are four motivations to why therapists engage in PP: personal problems, personal growth, self-care, and therapist skill development. Furthermore, associated with these motivations are five outcomes that may result from engaging in PP: personal development/well-being, enhanced self-awareness, and improvement in interpersonal, reflective, and conceptual/technical skills (i.e. the ability to use treatment-specific knowledge and techniques with patients). So far, less attention has been paid to therapist self-application of treatment techniques in PP models, although such self-care strategies may influence therapist effectiveness, which influences patient outcome. For example, research suggests that therapist qualities such as resilience, mindfulness, self-confidence and capacity for self-reflection and self-awareness are associated with positive patient outcome (Bennett-Levy, 2019), and it is likely that such qualities are malleable by self-applying treatment techniques.

The purpose of the present study was to explore the use of specific cognitive behavioural therapy (CBT) techniques for self-care among practising therapists, either when they themselves had experienced mental health problems, or for preventing such problems to occur. In addition, to get a fuller picture of therapists' self-care using CBT techniques, perceived usefulness of the techniques was explored. Thus, the study aimed to expand the self-care literature to these types of strategies and inspire further development of PP models.

\section{Method}

\section{Participants}

Participants were psychologists, social workers, nurses and an additional category of 'other' therapists (e.g. physicians, psychiatric aides, occupational therapists), all of whom were practising CBT $(n=228)$. Participants either had membership in one of the two largest multidisciplinary interest groups for CBT in Sweden (the Swedish Association of Cognitive and Behavioral Therapies and the Swedish Association of Behavior Therapy) or were employed within the child and adolescent psychiatric health care services in the city of Linköping, Sweden. For participant characteristics, see Table 1. A majority (76.3\%) of participants had undergone previous personal therapy based on CBT, which may be due to the fact that in Sweden personal therapy has been, and at some universities continues to be, a mandatory part of the training of psychologists, and often an admission requirement for training programmes of therapists at the master's level. 
Table 1. Participant characteristics

\begin{tabular}{|c|c|}
\hline & $n(\%)$ \\
\hline Gender, females & $187(82.0)$ \\
\hline Age in years, mean ( $S D$; range) & $50.2(10.4 ; 27-77)$ \\
\hline \multicolumn{2}{|l|}{ Profession } \\
\hline Psychologist & $101(44.3)$ \\
\hline Social worker & $68(29.8)$ \\
\hline Nurse & $24(10.5)$ \\
\hline Other & $35(15.3)$ \\
\hline Time since graduation in years, mean ( $S D$; range) & $20.5(11.4 ; 1-49)$ \\
\hline Clinical experience in years, mean (SD; range) & $17.1(10.7 ; 1-45)$ \\
\hline \multicolumn{2}{|l|}{ Level of training in $\mathrm{CBT}^{\mathrm{a}}$} \\
\hline Supervisor ${ }^{b}$ & $31(13.8)$ \\
\hline Master's ${ }^{c}$ & $80(35.6)$ \\
\hline Basic $^{d}$ & $109(48.4)$ \\
\hline Less than basic & $5(2.2)$ \\
\hline Previous experience of personal therapy based on CBT & $174(76.3)$ \\
\hline
\end{tabular}

CBT, cognitive behavioural therapy. ${ }^{a}$ Missing, $n=3$. ${ }^{b}$ Equivalent to 0.5 -year full-time studies; supervisor level requires previously attained master's level. 'Equivalent to 1.5 -year full-time studies; master's level requires previously attained basic level. ${ }^{\mathrm{d} E q u i v a l e n t}$ to 1 -year full-time studies.

\section{Assessment}

Data were collected using a web-based survey (LimeSurvey, version 2.06+), hosted by the Department of Behavioral Sciences and Learning at Linköping University, Linköping, Sweden. In a pilot phase, three individuals not participating in the present study responded to the survey while simultaneously being interviewed about the comprehensibility and relevance of the items. As a result, instructions were improved and some items were rephrased. The survey items covered characteristics of therapists and their use, and perceived usefulness, of 13 CBT techniques for preventative or corrective self-care of own mental health problems. In addition, the survey included items on therapist attitudes towards self-care, and participants were provided with the opportunity to leave comments and make reflections on the subject in a free text format. The sample of CBT techniques was selected based on a Swedish textbook on CBT (Linton and Flink, 2010), and the CORE Competence Framework for Cognitive and Behavioural Therapy (Roth and Pilling, 2008). The techniques were: self-assessment (e.g. of thoughts, sleep pattern), self-report measures (e.g. the Patient Health Questionnaire-9 for depressive symptoms; Kroenke et al., 2001), cognitive techniques (e.g. cognitive restructuring, defusion), worry control (e.g. worry postponement), behavioural experiments (to challenge beliefs), expressive writing (e.g. to process a difficult experience), exposure to feared stimuli (e.g. social situations), behavioural activation (e.g. to counteract depressive symptoms), applied relaxation (e.g. to relieve distress), stress management (e.g. to prioritize among activities), sleep management (e.g. sleep hygiene, stimulus control), mindfulness (e.g. to increase selfregulation) and communication skills (e.g. assertiveness). An example item of use of techniques is: 'Have you practised exposure on yourself, for example, of specific phobia, social anxiety, etc.?'. Use of techniques was rated Yes or No. An example item of perceived usefulness of the techniques is: 'Exposure has been useful'. Usefulness was rated on a Likert scale, ranging from 0 (not at all) to 4 (very much). Therapists were invited to participate by email, which included a link to the survey. The survey took about five minutes to complete, and therapists responded to it anonymously. The survey was accessible during a 2-week period in 2017. 


\section{Data analysis}

Data were analysed using the SPSS (version 26, SPSS Inc., Chicago, IL, USA). Descriptive statistics were calculated. Examination of the data showed that non-parametric analyses were appropriate. Given the explorative approach of the present study, associations of the use, and perceived usefulness, of CBT techniques for self-care with several participant characteristics were investigated. Associations of the use $(\mathrm{Yes} / \mathrm{No})$ of techniques with gender, profession (with psychologists, social workers, nurses and other therapists as levels), level of training in CBT (with supervisor, master's, basic, and less than basic as levels), and previous experience of personal therapy based on CBT were analysed using chi-square tests and Fisher's exact test. To investigate what level of the variables with four levels contributed to the overall association that the chi-square statistic measures the standardized residuals were inspected. The standardized residual is the error between what the model predicts (the expected frequency) and the observed data (the observed frequency).

Associations of perceived usefulness (rated on a 0- to 4-point scale) of CBT techniques with gender and previous personal CBT were analysed using Mann-Whitney tests. Associations of usefulness of techniques with age, years since graduation and clinical experience were analysed using Spearman's correlation coefficient $r_{\mathrm{s}}$. Associations of usefulness of techniques with profession and level of training in CBT were analysed using Kruskal-Wallis tests. Effect sizes for Mann-Whitney and Kruskal-Wallis tests were calculated as $r=z / \sqrt{ } N$, where $z=$ the standardized test statistic and $N=$ total sample size on which $z$ is based.

\section{Results}

\section{Use of CBT techniques for self-care}

There was a high prevalence of the use of CBT techniques for self-care among practising therapists, either when they had experienced mental health problems or for the prevention of such problems (Table 2). On average, participants had used 8.52 techniques $(S D=2.57$, range $=2-13$ ). The three most used techniques were mindfulness (95.6\%), cognitive techniques $(87.3 \%)$ and stress management (86.4\%). Ten of the 13 surveyed techniques had been used by more than $50 \%$ of participants. There were no differences between females and males on the use of techniques, except for mindfulness $\left[\chi^{2}(1)=8.17, p=.014\right]$ and cognitive techniques $\left[\chi^{2}(1)=5.30, p=.030\right]$; the odds of having used these techniques were 5.52 and 2.75 times higher, respectively, if the participant was a female than if being a male. Regarding associations of the use of techniques with profession, more psychologists than expected had not used mindfulness or stress management for self-care: $\chi^{2}(3)=8.75, p=.014$ and $\chi^{2}(3)=8.31, p=.035$, respectively. There were also differences between participants according to profession for worry control $\left[\chi^{2}(3)=11.36, p=.009\right]$ and applied relaxation $\left[\chi^{2}(3)=8.57, p=.035\right]$; however, it was not clear what level of profession (i.e. psychologists, social workers, nurses, other therapists) contributed to the overall associations. For the remainder of techniques, there were no differences based on profession. Furthermore, there were no differences between participants on the use of techniques according to level of training in CBT, except for cognitive techniques $\left[\chi^{2}(3)=11.22, p=.008\right]$, where more therapists with basic training than expected, and less master's level-therapists than expected, had not used the technique. Concerning differences between participants on the use of techniques according to previous experience of personal therapy based on CBT, there were differences for sleep management $\left[\chi^{2}(1)=5.88, p=.022\right]$, worry control $\left[\chi^{2}(1)=4.59\right.$, $p=.032]$ and behavioral experiments $\left[\chi^{2}(1)=5.36, p=.021\right]$; for these techniques, participants without personal CBT were more likely not to have used them. For the remainder of techniques, there were no differences based on personal CBT. 


\section{Perceived usefulness of CBT techniques for self-care}

Overall, participants perceived CBT techniques as useful for self-care (see Table 2). The average on the 0 - to 4-point usefulness scale was $2.79(S D=0.35)$, and the three techniques perceived as most useful were exposure (3.18), mindfulness (3.05) and behavioural activation (3.04). Perceived usefulness of techniques did not differ between males and females, except for mindfulness $(U=1968.00, p=.001, r=-.23)$, stress management $(U=1911.00, p=.041, r=-.15)$ and applied relaxation $(U=842.00, p=.015, r=-.21)$; these techniques were perceived as more useful by females than males. Usefulness of the techniques did not differ between participants according to therapist age, years since graduation or clinical experience, except for the usefulness of behavioural activation, which was negatively associated with years since graduation (Spearman's $r_{\mathrm{s}}=-.17, p=.039$ ). Usefulness did not differ based on previous personal CBT, except for exposure $(U=2068.00, p=.029, r=-.17)$ and communication skills $(U=1109.00, p=.034, r=-.18)$; these techniques were perceived as more useful by participants with previous personal CBT. Usefulness did not differ between participants according to profession, except for self-report measures $[H(3)=7.84, p=.049]$, where pairwise comparisons showed that nurses perceived self-report measures as less useful than other therapists $(p=.049, r=-.48$ ). Furthermore, usefulness did not differ between participants according to level of training in CBT, except for mindfulness $[H(3)=8.15$, $p=.043$ ], where therapists with less than basic training perceived mindfulness as less useful than both basic-level and master's-level therapists: $p=.037, r=-.26$ and $p=.026, r=-.32$, respectively. Perceived usefulness also differed between participants according to level of training in CBT for cognitive techniques $[H(3)=9.09, p=.028]$; however, no pairwise comparisons were significant, meaning it was not possible to discern what level of training in CBT (i.e. supervisor, master's, basic, and less than basic) contributed to the overall association.

\section{Therapist attitudes towards CBT techniques for self-care}

On survey items on therapist attitudes towards self-care, almost all participants (98.7\%) responded that it was a good idea to self-apply CBT techniques to prevent or treat mental health problems of their own. A similar proportion (97.4\%) considered it useful to have selfapplied a technique before they introduced it to patients in treatment. A large proportion of participants $(89.5 \%)$ believed that it was important for them to regularly practise CBT techniques on themselves; however, a smaller proportion (36.4\%) reported that they keep updated on research on self-care strategies. Using the free text option in the survey, several participants commented that it was beneficial for their practice to have self-applied CBT techniques; for example, personal experience of the techniques was considered important in terms of self-confidence and credibility as a therapist, and for the working alliance with the patient. Also, personal experience was considered important for guiding patients in the implementation of techniques and understanding the difficulties that may arise in the process.

\section{Discussion}

The purpose of the present study was to explore the use, and perceived usefulness, of CBT techniques for self-care among practising therapists, either when they had experienced mental health problems or for the prevention of such problems. Results showed that therapists had applied CBT techniques on themselves to a large extent, as all therapists had used at least two techniques, with an average of almost nine techniques. With few exceptions, there were no differences between participants in the use of techniques according to gender, profession, level of training in CBT, and previous experience of personal therapy based on CBT. It should be noted that although the level of significance was not corrected for multiple testing, there were 
few differences between participants in the use of techniques based on therapist characteristics; this indicates that the use of the techniques was universal in the present sample of therapists. Of course, the high prevalence among the therapists of the use of CBT techniques to prevent or treat own mental health problems is very encouraging.

Participants perceived the CBT techniques as useful for self-care. With few exceptions, and similar to use of techniques, there were no differences in perceived usefulness of techniques based on gender, age, profession, years since graduation, clinical experience, level of training in CBT, and previous personal CBT. Again, although the level of significance was not corrected for multiple testing, there were few differences between participants in perceived usefulness of the techniques based on therapist characteristics, indicating the universal appreciation of the techniques in this sample of therapists. Finally, almost all therapists believed that it was appropriate to self-apply techniques and to practise them regularly, both for their own sake and for the benefit of their patients in treatment. This suggests the potential value of including self-care in the form of self-application of treatment techniques in the training and professional development of therapists.

As mentioned previously, the literature on self-care of therapists mainly focuses on preventative self-care strategies, such as maintaining a balance between private and professional lives, exercising healthy dietary and physical activity habits, and receiving supervision (Barnett et al., 2007; Barnett and Cooper, 2009), and such corrective self-care strategies as undergoing personal therapy (Orlinsky et al., 2011), and more recently attending meditation programmes (Bennett-Levy, 2019). Published research on corrective strategies for self-care involving the self-application of specific treatment techniques is lacking, making it difficult to identify findings to compare the results of the present study with. For example, in the aforementioned survey of psychologists in the USA (American Psychological Association, 2010), self-application of treatment techniques was not among the ten most frequently reported self-care strategies. One reason could be that most studies on self-care involved samples of therapists with other therapeutic orientations than CBT (or where CBT was part of a mixture of orientations), and that other orientations, such as psychodynamic therapy, may focus more on 'relationship-dependent' techniques (Blagys and Hilsenroth, 2000; Boterhoven de Haan and Lee, 2013) such as transference, and less on techniques that are not as relationship dependent, e.g. cognitive techniques, which may be required for applying treatment techniques on oneself. However, to what extent techniques from other therapeutic orientations than CBT may be used for self-care remains to be investigated.

Therapist self-care, including the self-application of treatment techniques, may be conceptualized as part of a PP model, such as the model developed by Bennett-Levy and Finlay-Jones (2018). Clearly, the four motivations to why therapists engage in PP according to this model, that is, personal problems, personal growth, self-care and therapist skill development overlap and interact with each other. For example, therapists may engage in PP to take care of personal problems and grow as people, and solving personal problems using self-care strategies may be associated with therapist skill by increasing therapist qualities such as resilience and self-confidence, which are associated with patient outcome (Bennett-Levy, 2019). Thus, self-care, including the self-application of treatment techniques, may be an important factor for therapist effectiveness, which calls for further development of PP models with respect to self-care, and future studies investigating associations between therapist mental health, self-care, effectiveness and patient outcome. To date such studies are lacking.

The present study had several limitations. First, no definition of 'mental health' was provided to participants, and the definitions of CBT techniques could have been more complete. Thus, participants' responses may have varied depending on how they perceived the survey items. Second and related, because of the lack of validated measures of the use, and perceived usefulness, of CBT techniques for self-care in therapists, the survey was developed for use in the present study and its psychometric properties are not known. Third, there was no division 
of prevention and treatment of mental health problems, so it is not known whether therapists used and perceived the usefulness of techniques in the context of prevention or treatment. Thus, it is conceivable that the high prevalence of techniques in this sample of therapists could be due to them using the techniques mostly for preventative purposes. Fourth, the frequency of the use of techniques is not known, only that the participants had used them at least once. Finally, the circumstance that almost half of the participants were psychologists who had undergone personal therapy as part of their training restricts the generalizability of the findings. However, profession or previous personal CBT did not influence the use or perceived usefulness of the techniques among the participants.

In summary, the present study is one of few studies exploring practising therapists' use, and perceived usefulness, of CBT techniques for self-care of mental health problems or for preventing such problems to occur. Results showed that the use of CBT techniques for self-care was highly prevalent among the participants, and that they perceived them as useful, irrespective of therapist characteristics such as gender, age, profession, years since graduation, clinical experience, level of training in CBT, or previous personal CBT. Arguably, self-care, including the self-application of treatment techniques, is an important factor for therapist effectiveness and thus patient outcome and deserves increased attention in further research. However, at present there are limited empirical data to support these associations. Therefore, future studies should continue to develop and validate PP models by investigating associations between therapist mental health, self-care, effectiveness, and patient outcome, using correlational and experimental designs.

Acknowledgements. We thank web master George Vlaescu at Linköping University, Linköping, Sweden for assistance.

Financial support. This work was supported in part by a professor's grant to Gerhard Andersson by the Linköping University, Linköping, Sweden.

Conflicts of interest. None.

Ethical statements. The authors have abided by the Ethical Principles of Psychologists and Code of Conduct as set out by the British Association for Behavioural and Cognitive Psychotherapies and the BPS. The institutional review board of the Department of Behavioural Sciences and Learning at Linköping University, Linköping, Sweden approved the study.

Key practice points

(1) Being aware of own health issues and their possible effects on patients, as well as maintaining fitness to practise, is an ethical obligation for therapists.

(2) Therapists should exercise self-care using a variety of both preventative and corrective strategies, including the self-application of treatment techniques, which are naturally accessible to therapists.

(3) Self-applying treatment techniques for self-care may benefit not only therapists themselves but also their patients.

\section{Further reading}

Barnett, J. E., \& Cooper, N. (2009). Creating a culture of self-care. Clinical Psychology: Science and Practice, 16, 16-20. https:// doi.org/10.1111/j.1468-2850.2009.01138.x

Bennett-Levy, J. (2019). Why therapists should walk the talk: the theoretical and empirical case for personal practice in therapist training and professional development. Journal of Behavior Therapy and Experimental Psychiatry, 62, 133-145. https://doi.org/10.1016/j.jbtep.2018.08.004

Bennett-Levy, J., \& Finlay-Jones, A. (2018). The role of personal practice in therapist skill development: a model to guide therapists, educators, supervisors and researchers. Cognitive Behaviour Therapy, 47, 185-205. https://doi.org/10.1080/ 16506073.2018.1434678 


\section{References}

American Psychological Association (2002). Ethical principles of psychologists and code of conduct. American Psychologist, 57, 1060-1073. https://doi.org/10.1037/0003-066X.57.12.1060

American Psychological Association (2010). Listening to our colleagues: 2009 Practice Survey - worries, wellness, and wisdom. Retrieved from: https://www.apa.org/practice/resources/assistance/acca-2010-convention.pdf

Barnett, J. E., Baker, E. K., Elman, N. S., \& Schoener, G. R. (2007). In pursuit of wellness: the self-care imperative. Professional Psychology: Research and Practice, 38, 603-612. https://doi.org/10.1037/0735-7028.38.6.603

Barnett, J. E., \& Cooper, N. (2009). Creating a culture of self-care. Clinical Psychology: Science and Practice, 16, 16-20. https:// doi.org/10.1111/j.1468-2850.2009.01138.x

Bennett-Levy, J. (2019). Why therapists should walk the talk: the theoretical and empirical case for personal practice in therapist training and professional development. Journal of Behavior Therapy and Experimental Psychiatry, 62, 133-145. https://doi.org/10.1016/j.jbtep.2018.08.004

Bennett-Levy, J., \& Finlay-Jones, A. (2018). The role of personal practice in therapist skill development: a model to guide therapists, educators, supervisors and researchers. Cognitive Behaviour Therapy, 47, 185-205. https://doi.org/10.1080/ 16506073.2018.1434678

Blagys, M. D., \& Hilsenroth, M. J. (2000). Distinctive features of short-term psychodynamic-interpersonal psychotherapy: a review of the comparative psychotherapy process literature. Clinical Psychology: Science and Practice, 7, 167-188. https:// doi.org/10.1093/clipsy.7.2.167

Boellinghaus, I., Jones, F. W., \& Hutton, J. (2013). Cultivating self-care and compassion in psychological therapists in training: the experience of practicing loving-kindness meditation. Training and Education in Professional Psychology, 7 , 267-277. https://doi.org/10.1037/a0033092

Boterhoven de Haan, K. L., \& Lee, C. W. (2013). Therapists' thoughts on therapy: clinicians' perceptions of the therapy processes that distinguish schema, cognitive behavioural and psychodynamic approaches. Psychotherapy Research, 24, 538-549. https://doi.org/10.1080/10503307.2013.861092

European Federation of Psychologists' Associations (2005). Meta-Code of Ethics. Retrieved from: http://ethics.efpa.eu/ metaand-model-code/meta-code/

Gilroy, P. J., Carroll, L., \& Murra, J. (2002). A preliminary survey of counseling psychologists' personal experience with depression and treatment. Professional Psychology: Research and Practice, 33, 402-407. https://doi.org/10.1037/07357028.33.4.402

Health and Care Professions Council (2015). The standards of proficiency for practitioner psychologists. Retrieved from: https:/www.hcpc-uk.org/standards/standards-of-proficiency/practitioner-psychologists/

Kroenke, K., Spitzer, R. L., \& Williams, J. B. (2001). The PHQ-9: validity of a brief depression severity measure. Journal of General Internal Medicine, 16, 606-613. https://doi.org/10.1046/j.1525-1497.2001.016009606.x

Linton, S. J., \& Flink, I. (2010). 12 Tools of CBT: From Theory to Practice [12 verktyg i KBT: från teori till färdighet]. Stockholm, Sweden: Natur och Kultur.

Lomas, T., Medina, J. C., Ivtzan, I., Rupprecht, S., \& Eiroa-Orosa, F. J. (2019). A systematic review and meta-analysis of the impact of mindfulness-based interventions on the well-being of healthcare professionals. Mindfulness, 10, $1193-1216$. https://doi.org/10.1007/s12671-018-1062-5

Mental Health Foundation (2016). Fundamental Facts about Mental Health 2016. London, UK: Mental Health Foundation.

Norcross, J. C. (2000). Psychotherapist self-care: practitioner-tested, research-informed strategies. Professional Psychology: Research and Practice, 31, 710-713. https://doi.org/10.1037110735-7028.31.6.710

Orlinsky, D. E., Schofield, M. J., Schroder, T., \& Kazantzis, N. (2011). Utilization of personal therapy by psychotherapists: a practice-friendly review and a new study. Journal of Clinical Psychology, 67, 828-842. https://doi.org/10.1002/jclp.20821

Roth, A. D., \& Pilling, S. (2008). Using an evidence-based methodology to identify the competences required to deliver effective cognitive and behavioural therapy for depression and anxiety disorders. Behavioural and Cognitive Psychotherapy, 36, 129-147. https://doi.org/10.1017/S1352465808004141

Simionato, G. K., \& Simpson, S. (2018). Personal risk factors associated with burnout among psychotherapists: a systematic review of the literature. Journal of Clinical Psychology, 74, 1431-1456. https://doi.org/10.1002/jclp.22615

Tay, S., Alcock, K., \& Scior, K. (2018). Mental health problems among clinical psychologists: stigma and its impact on disclosure and help-seeking. Journal of Clinical Psychology, 74, 1545-1555. https://doi.org/10.1002/jclp.22614

Cite this article: Andersson G, Björklind A, Bennett-Levy J, and Bohman B. Use, and perceived usefulness, of cognitive behavioural therapy techniques for self-care among therapists. The Cognitive Behaviour Therapist. https://doi.org/10.1017/ S1754470X20000483 